\title{
CAPILARIOSE HEPÁTICA EM CÃES E GATOS: 15 CASOS
}

\author{
HEPATIC CAPILLARIOSIS IN DOGS AND CATS: 15 CASES
}

\author{
Mareia Regina da Silva Ilha ${ }^{1}$ Cláudio Severo Lombardo de Barros ${ }^{2}$
}

RESUMO

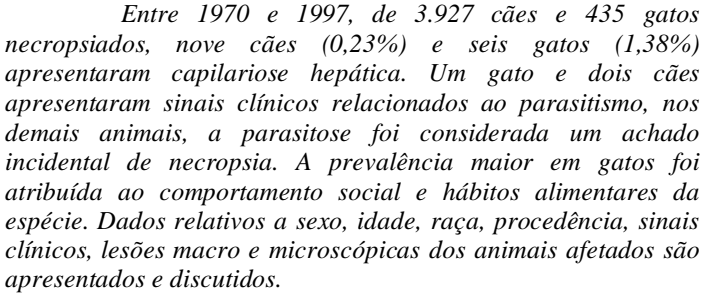

Entre 1970 e 1997, de 3.927 cães e 435 gatos necropsiados, nove cães $(0,23 \%)$ e seis gatos $(1,38 \%)$ apresentaram capilariose hepática. Um gato $e$ dois cães apresentaram sinais clínicos relacionados ao parasitismo, nos demais animais, a parasitose foi considerada um achado incidental de necropsia. A prevalência maior em gatos foi atribuída ao comportamento social e hábitos alimentares da espécie. Dados relativos a sexo, idade, raça, procedência, sinais clínicos, lesões macro e microscópicas dos animais afetados são apresentados e discutidos.

Palavras-chave: Capilaria hepática, parasitas, doenças de cães e gatos, patologia.

SUMMARY

Out of 3,927 dogs and 435 cats necropsied from 1970 to 1997 , nine dogs $(0.23 \%)$ and six cats $(1.38 \%)$ had hepatic capilariosis. One cal and one dog presented clinical signs related to the parasitism; in ali the other affected animals the parasitism was considered an incidental necropsy fmding. The higher prevalence in cats was attributed to the social behavior and alimentary habits of the species. Data on sex, age, breed, origin, clinical signs, gross and histopathological findings of the affected animais are presented and discussed.

Key words: Copularia hepática, parasites, diseases of dogs and cats, pathology.

\section{INTRODUÇÃO}

Capinaria hepatica foi descrita pela primeira vez em 1850, no fígado de um rato (CROSS, 1998). Desde então, tem sido descrita em muitas espécies de mamíferos, incluindo o homem (CHOE et al., 1993; KOHATSU et al., 1995). É considerado um parasita primariamente de roedores (FREEMAN \& WRIGHT, 1960; CROWELL et al., 1978) tendo sido relatado em Rattus norvegicus, $R$. rattus. Mus musculos e vários outros roedores silvestres (FREEMAN \& WRIGHT, 1960; SOLOMON \& HANDLEY, 1971; FARHANG-AZAD, 1977a; CONLOGUE et al., 1979). Outras espécies afetadas incluem coiotes (Canis latrans), gambás (Spilogale putorius) (CROWELL et al., 1978), cães, gatos, porcos, coelhos, lebres, ungulados e primatas nãohumanos (SOULSBY, 1968; STOKES, 1973; CROSS, 1998). Ovos de $\boldsymbol{C}$. hepática também foram encontrados em insetos (SOLOMON \& HANDLEY, 1971; FARHANG-AZAD, 1977b).

No Brasil, o parasitismo por $\boldsymbol{C}$. hepática foi descrito em ratazanas (Ratttus norvegicus) (ARAÚJO, 1967; GALVÃO, 1981), rato dos telhados (R. rattus) (CHDEFFI et al., 1981), cães (VIANNA, 1954; SANTOS \& BARROS, 1973; SILVEIRA et al., 1975), gatos (SANTOS \& BARROS, 1973), caxinguelê (Sciurus aestuans) (Freitas \& Lent, 1936 apud VIANNA, 1954), caititu (Tayassu tajacu) (MANDORINO \& REBOUÇAS, 1991) e graxaim-do-campo (Dusicyon gymnocercus) (RUAS, 1999 - Informe Verbal).

O ciclo de $\boldsymbol{C}$. hepatica é direto. A infecção se dá pela ingestão de ovos embrionados que eclodem no ceco, liberando larvas que migram, pelo sistema porta, até o fígado onde são encontrados vermes adultos e ovos (GALVÃO, 1981). A liberação dos ovos do fígado ocorre após a morte e decomposição do hospedeiro, ou pela eliminação através das fezes de animais que tenham ingerido fígado (predação ou

${ }^{1}$ Estudante do $11^{\circ}$ semestre de Medicina Veterinária da Universidade Federal de Santa Maria (UFSM), bolsista de Iniciação Científica do CNPq.

${ }^{2}$ Médico Veterinário, PhD., Professor Titular do Departamento de Patologia da UFSM, 97105-900, Santa Maria - RS. E-mail: cslb@sm.conex.com.br. Autor para correspondência. 
canibalismo) de animal parasitado (FARHAZGAZAD \& SCHLITTER, 1978). Os ovos no meio ambiente necessitam de 28 a 30 dias para se tomarem embrionados e infectantes (LUTTERMOSER, 1938; GALVÃO， 1981), requerendo altas taxas de oxigênio e umidade no solo (SOULSBY, 1968).

Este trabalho descreve a prevalência, o significado clínico, os dados epidemiológicos, os sinais clínicos e as lesões macro e microscópicas do parasitismo por $\boldsymbol{C}$. hepatica em cães e gatos necropsiados no Setor de Patologia Veterinária da Universidade Federal de Santa Maria.

\section{MATERIAL E MÉTODO}

Os arquivos do Setor de Patologia Veterinária da Universidade Federal de Santa Maria, do período entre 1970 a 1997, foram revisados à procura de cães e gatos parasitados pelo nematódeo C. hepatica. Foram anotados o número de animais necropsiados e o número de animais parasitados. Nestes, foram relacionados idade, sexo, raça e procedência. Os sinais clínicos, lesões macro e microscópicas foram revisados para se determinar o significado clínico do parasitismo nesses animais. Macroscopicamente, as lesões foram classificadas pela sua distribuição em difusa e multifocal e, microscopicamente, segundo sua intensidade, em leves, moderadas e acentuadas. De acordo com a distribuição e intensidade, as lesões foram classificadas como Gl (difusa com lesões acentuadas), $G 2$ (difusa com lesões moderadas), G3 (multifocal com lesões moderadas) e $G 4$ (multifocal com lesões leves).

\section{RESULTADOS}

No período estudado, foram necropsiados 3.927 cães e 435 gatos, dos quais 9 cães $(0,23 \%)$ e 6 gatos $(1,38 \%)$ apresentaram parasitismo por $\boldsymbol{C}$. hepatica. Os dados (idade, sexo, raça, procedência e grau de lesão) dos cães e gatos parasitados constam das tabelas 1 e 2 . Três animais apresentaram sinais clínicos relacionados à doença hepática. O cão 12 apresentou sinais clínicos de distúrbios nervosos acompanhados de vômitos, que iniciaram 1 mês antes da morte e o cão 8 apresentou, durante oito dias, apatia, anorexia, vômitos, dor abdominal, congestão conjuntival e fezes amareladas e fétidas. O gato 3 apresentou dor abdominal, icterícia, salivação, apatia e decúbito permanente seguido de morte. O cão 15 estava caquético, apesar de se alimentar bem. Esse animal foi submetido à eutanásia por apresentar carcinoma de células escamosas na pele.
Tabela 1 - Capilariose hepática em cães.

\begin{tabular}{ccccccc}
$N^{\circ}$ & Raça & $\begin{array}{c}\text { Idade } \\
\text { (anos) }\end{array}$ & Sexo & Procedência & Significado $\begin{array}{c}\text { Grau de } \\
\text { Lesão }\end{array}$ \\
\hline 07 & Srd & 4 & Nc & P & I & G4 \\
08 & Srd & 5 & Nc & Nc & Rdc & Gl \\
09 & Srd & 7 & F & P & I & G3 \\
10 & Srd & 5 & F & R & I & G4 \\
11 & Srd & 7 & F & R & I & G3 \\
12 & Srd & 8 & F & P & Rdc & G1 \\
13 & Srd & Adulto & M & R & I & G2 \\
14 & Dálmata & 4 & M & P & I & G4 \\
15 & Srd & 10 & F & R & I & G1 \\
& & & & & & \\
\hline
\end{tabular}

Srd = sem raça definida;

$\mathrm{Nc}=$ não consta;

$\mathrm{F}=$ fêmea;

$\mathrm{M}=$ macho;

$\mathrm{R}=$ Biotério (cão de rua);

$\mathrm{P}=$ cão com proprietário;

I = achado incidental;

$\mathrm{Rdc}=$ relacionado com doença clínica.

Nas necropsias, foram observadas lesões hepáticas de intensidades variadas. Lesões difusas eram representadas por fígado firme, pálido, levemente aumentado de volume e com superfície capsular e de corte irregular, finamente granular e de aspecto reticulado conferido por linhas amareladas que se entrecruzavam no parênquima do órgão (Figura 1). Nas lesões multifocais, ocorriam apenas pontos amarelados distribuídos aleatoriamente na superfície natural e de corte.

Histologicamente, as lesões leves consistiam de poucos agregados multifocais de ovos de C. hepatica sem resposta inflamatória ou acompanhados de leve infiltrado inflamatório mononuclear com raros eosinófilos e mínima proliferação de fíbroblastos ao redor dos ovos. Nas lesões moderadas, havia vários aglomerados de ovos com presença de cortes transversais e longitudinais de parasitas adultos, alguns já em processo de degeneração, cercados por infiltrado inflamatório mononuclear com eosinófilos (Figura 2) e presença de células gigantes. As lesões acentuadas tinham o mesmo padrão das moderadas, porém eram mais extensas, tinham menor número de eosinófilos, maior número de ovos e degeneração gordurosa de hepatócitos próximos aos parasitas e ovos. Calcificação de ovos foi observada em alguns casos.

\section{DISCUSSÃ̃O}

O diagnóstico nesses 15 casos foi baseado na presença de cortes transversais e longitudinais dos parasitas adultos e ovos típicos de Copularia

Ciência Rural, v. 30, n. 4, 2000. 
Tabela 2 - Capilariose hepática em gatos*.

\begin{tabular}{ccccc}
\hline$N^{\circ}$ & $\begin{array}{c}\text { Idade } \\
\text { (anos) }\end{array}$ & Sexo & Significado & $\begin{array}{c}\text { Grau de } \\
\text { Lesão }\end{array}$ \\
\hline 01 & Adulto & M & I & G4 \\
02 & Adulto & M & I & G2 \\
03 & 1 & M & Rdc & G1 \\
04 & 4 & M & I & G4 \\
05 & 2 & F & I & G2 \\
06 & $<1$ & M & I & G2 \\
\hline
\end{tabular}

*Todos animais com proprietário e mestiços.

$\mathrm{M}=$ macho;

$\mathrm{F}=$ fềmea;

I= achado incidental;

$\mathrm{Rdc}=$ relacionado com doença clínica.

hepatica localizados no parênquima hepático. Os ovos de C. hepatica medem de $50-68$ por $28-35 / \mu \mathrm{m}$, têm forma de barril e casca espessa, dupla, com tampões bipolares e pequenos poros na camada externa da casca, dando a aparência de estriações (SOULSBY, 1968; SOLOMON \& HANDLEY, 1971;CROSS,1998).

As taxas de prevalência do parasitismo por C. hepatica em cães e gatos foram de $0,23 \%$ e $1,38 \%$ respectivamente. Essas taxas são menores quando comparadas às encontradas em algumas espécies de roedores como ratazanas (Rattus norvegicus). Nesses, as taxas variam de 56,5 a 89,3\% (FREEMAN \& WRIGHT, 1960; ARAÚJO, 1967; SOLOMON \& HANDLEY, 1971; FARHANG-AZAD, 1977b). A alta prevalência e baixa intensidade de infecção em $R$. norvegicus sugerem que essa espécie seja o hospedeiro primário

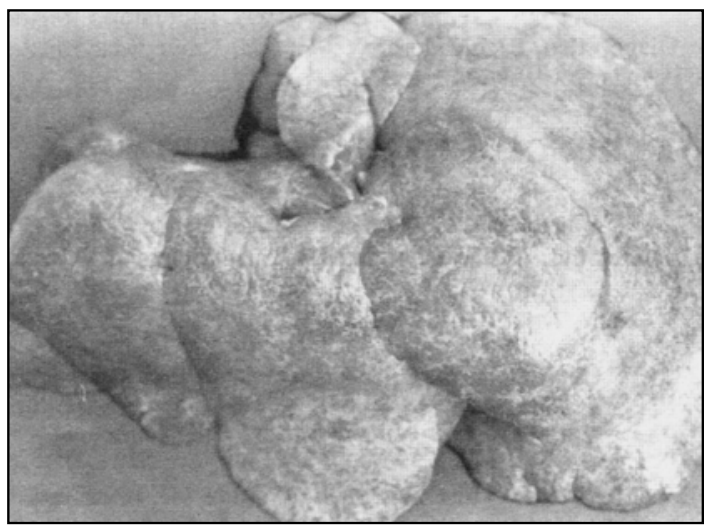

Figura 1 - Capilariose hepática. Macroscopia do fígado. Cão 15. O fígado está mais pálido, levemente aumentado de volume e a superfície natural é levemente granular com aspecto reticulado causado por linhas mais claras que se entrecruzam no parênquima. de $\boldsymbol{C}$. hepatica. O ciclo de renovação rápido da população de ratos contribui para a rápida liberação de grande número de ovos no meio ambiente e as altas taxas de nascimento fornecem número suficiente de hospedeiros suscetíveis para completar o ciclo do parasita (FARHANG-AZAD, 1977a). Em roedores em geral, dependendo da espécie, as taxas de prevalência variam de 0,5 a $89,3 \%$, (FREEMAN \& WRIGHT, 1960; ARAÚJO, 1967; SOLOMON \& HANDLEY, 1971; FARHANG-AZAD \& SCHLITTER, 1978). Prevalências maiores já foram observadas em outros carnívoros. Em uma pesquisa com 216 cães nos Estados Unidos, foram observados 3 casos (WRIGHT, 1930) e no Japão, de 65 cães examinados, $8 \%$ apresentavam o parasita (Itagaki, 1929 apud WRIGHT, 1930). CROWELL et al. (1978) observaram a prevalência de $30 \%$ do parasitismo por C. hepatica em coiotes na América do Norte e RUAS (1999 - Informe Verbal), no Rio Grande do Sul, verificou que, de 32 graxains-docampo examinados, cinco apresentavam parasitismo por C. hepatica.

A prevalência maior em gatos pode ser explicada pelo comportamento social e alimentar dessa espécie. Gatos possuem áreas domésticas definidas por onde circulam, com trilhas a partir dessa área central para áreas especiais onde desempenham determinadas atividades, como caça, descanso ou excreção (BEAVER, 1992). Sendo assim, os gatos, mesmo os com domicílio fixo, têm acesso a outros ambientes. Além disso, estudos em gatos selvagens confirmam que camundongos e ratos estão entre as presas preferidas dos gatos (BEAVER, 1992). A predação, nesse caso, contribui para a disseminação dos ovos no ambiente dos felinos, uma vez que a eliminação dos ovos pelas fezes desses

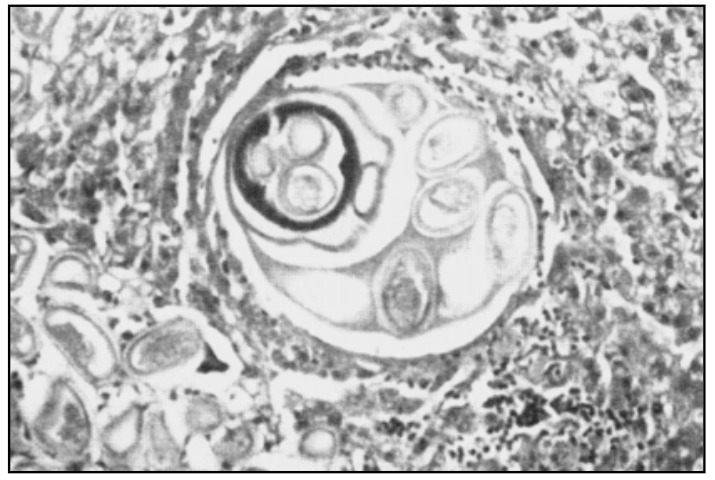

Figura 2 - Capilariose hepática. Histopalogia do fígado. Cão 15. Ao centro se observa exemplar fêmea de Capillaria hepatica contendo ovos em seu interior. Abaixo e à esquerda, há acúmulo de ovos com tampões bipolares no parênquima hepático. Observe também infiltrado inflamatório mononuclear ao redor dos ovos e do parasita. 
animais perdura por três dias (SHORB, 1931). Fato semelhante, observado em outras espécies de carnívoros, também foi explicado pelas diferenças comportamentais entre as espécies. Assim, a alta prevalência encontrada em coiotes comparada com a inexistência do parasitismo em raposas do mesmo habitat foi atribuída, em parte, às diferenças entre os hábitos alimentares e de defecação nessas espécies (CROWELL et al., 1978). A ocorrência da capilariose em cães de rua pode estar relacionada aos hábitos alimentares pouco seletivos desses animais, com inclusão de carcaças de roedores na dieta (CONLOGUE et al., 1979). Cães de rua, ou os que têm acesso eventual às ruas, têm maiores possibilidades de se infectarem quando comparados a animais que frequentam um único ambiente. $\mathrm{O}$ parasitismo em cães de rua já foi observado anteriormente (VIANNA, 1954; AFIP, 1994). Assim, as espécies que funcionam como hospedeiros secundários são envolvidas quando seus hábitos as colocam em contato com os focos de infecção primária (SOLOMON \& HANDLEY, 1971). O hábito de predação em animais domésticos de rua contribui para disseminação de ovos (CONLOGUE et al., 1979), constituindo fontes de infecção para eles, para outros animais e para o homem.

Neste estudo, verificou-se que cães e gatos, sem raça definida, apresentaram maior prevalência ao parasitismo. Esse aspecto pode ser justificado pelo fato dessa categoria de animais ter sido a mais frequentemente necropsiada durante o período de 1976 a 1997. A infecção foi observada nas diferentes faixas etárias, à semelhança do que ocorre em roedores e humanos. Entretanto, em roedores, a frequência de infecção aumenta com a idade (CHTEFFI et al., 1981) e nos humanos sua ocorrência parece estar mais relacionada a crianças com idade inferior a dez anos (KOHATSU, 1995).

$\mathrm{Na}$ maioria dos casos neste estudo, a capilariose hepática foi um achado incidental de necropsia. Sinais clínicos como anorexia, vómitos, diarreia e icterícia, apresentados por dois animais, são manifestações de doença hepática em pequenos animais (CENTER, 1995). A dor abdominal observada nesses dois animais é também um sinal clínico observado em humanos parasitados por $\boldsymbol{C}$. hepatica (KOHATSU et al., 1995). Os distúrbios nervosos apresentados por um dos cães foram atribuídos a encefalopatia hepática. Animais com doença hepática difusa e acentuada podem desenvolver encefalopatia hepática caracterizada clinicamente por distúrbios neurológicos variados (TABOADA \& DIMSKI, 1995). Salivação, observada em um gato, é um sinal clínico comum em gatos com doença hepática e pode ser atribuída à encefalopatia hepática ou a náusea (CENTER, 1995). Sinais clínicos como anorexia, vómito, diarréia e mudança de comportamento em cães com capilariose hepática também foram observados por outros autores (VIANNA, 1954; SILVEIRAS et al., 1975).

Algumas particularidades da histopatologia do fígado dos animais deste estudo devem ser mencionadas. Quando somente ovos eram observados, havia presença de raros eosinófilos, além do infiltrado inflamatório mononuclear, células gigantes e calcificação de ovos. Quando eram encontrados parasitas adultos, ocorria um número consideravelmente maior de eosinófilos. Situação semelhantetem sido observada por outros autores (VIANNA, 1954; STOKES, 1973; SILVEIRA et al., 1975; AFIP 1994; KOHATSU et al., 1995). Também em ratos sacrificados aos 28 dias pósinfecção, têm sido relatados achados semelhantes (BORUCINSKA $\boldsymbol{e t}$ al., 1997). Vermes adultos e ovos eram vistos rodeados por eosinófilos, células gigantes, células epitelióides, plasmócitos e linfócitos. Nos animais sacrificados aos 184 dias pós-infecção, havia agregados hepáticos de tamanho variável de ovos de $\boldsymbol{C}$. hepatica circundados por leve fíbrose. Quando o número de ovos era pequeno, esses eram parcialmente mineralizados e fagocitados por células gigantes.

$\mathrm{Na}$ grande maioria dos roedores, as infecções por C. hepatica variam entre leves a acentuadas, mas nunca ocorrem associadas à doença clínica (CHIEFFI $\boldsymbol{e t}$ al., 1981). Neste estudo, pelo menos em três animais que apresentaram sinais clínicos de doença hepática associados a lesões hepáticas acentuadas e difusas, sugere-se que a parasitose esteja relacionada com a doença clínica.

\section{INFORME VERBAL}

RUAS, Jerônimo Lopes, 1999. Laboratório Regional de Diagnóstico, Faculdade de Veterinária, Universidade Federal de Pelotas, 96010-900 Pelotas, RS.

\section{REFERÊNCIAS BIBLIOGRÁFICAS}

AFIP. Wednesday slide conference. Conference $n^{9} 6$ de 19 de outubro de 1994. Washington, DC : Department of Veterinary Pathology, Anned Forces Instituto of Pathology, 1994. p.2-4. Mimeografado.

ARAÚJO, P. Helmintos de Rattus norvegicus (Berkenhout, 1769) da cidade de São Paulo. Rev Fac Farm Bioquím S Paulo, v.5, n.1, p.141-159,1967.

BEAVER, B.V. Feline behavior: A guide for veterinarians. Philadelphia : Saunders, 1992. 276p.

BORUCINSKA, J.D., van KRUDMINGEN, H.J., CAIRÁ, J.N., et al.. Clinicopathological features and histopathology of experimental hepatic capiliariasis in muskrats (Ondrata zibethicus). J Wildl Dis, v.33, n.1, p.122-130, 1997. 
CENTER, S.A. Pathophysiology, laboratory diagnosis, and diseases of the liver. In: ETTINGER, S.J., FELDMAN, E.C Textbook of veterinary internal medicine. 4 ed. Philadelphia : Sauders, 1995. Cap.106, p.1261-1312.

CHIEFFI, P.P., DIAS, R.M.D.S., MANGINI, A.C.S., $\boldsymbol{e t}$ al. Capilaria hepatica (BANCROFT, 1893), em murídeos capturados no município de São Paulo, SP, Brasil. Rev Inst Med Trop São Paulo, v.23, n.4, p.143-146,1981.

CHOE, G., LEE, H.S., SEO, J.K., et al. Hepatic capiliariasis: first case report in the republic of Korea. Am J Trop Med Hyg, v.48, n.5, p.610-625,1993.

CONlOGUE, G., FOREYT, W., ADESS, M., LEVINE, H. Capilaria hepatica (Bancroft) in select rat populations of Hartford, Connecticut, wiht possible public health implications. J Parasitology, v.65, n.l, p.105-108,1979.

CROSS, J.H. Capiliariosis. In: PALMER, S.R., SOULSBY, L., SIMPSON, I.H. Zoonoses. Oxford: University, 1998. Cap.57,p.773-781.

CROWELL, W.A., KLEI, T.R., HALL, D.I. Capiliaria hepatica infection in coyotes of Louisiana. J Am Vet Med Assoe, v.173,n.9,p.1171-1172,1978.

FARHANG-AZAD, A. Ecology of Capiliaria hepatica (Bancroft 1893) (Nematodea). I. Dinamics of infection among Norway rat populations of the Baltimore Zoo, Baltimore, Maryland. J Parasitology, v.63, n.1, p.117-122,1977a.

FARHANG-AZAD, A. Ecology of Capilaria hepatica (Bancroft 1893) (Nematodea). II. Egg-releasing mechanisms and transmission. J Parasitology, v.63, n.4, p.701-706, 1977b.

FARHANG-AZAD, A., SCHLITTER, D.A. Capilaria hepatica in small mammals coilected from Schoa Province, Ethiopia. J Wildl Dis, v. 14, n.3, p.358-361,1978.

FREEMAN, R.S., WRIGHT, K.A. Factors concemed with the epizootiology of Capiliaria hepatica (BANCROFT, 1893) (NEMATODA) in a population of Peromyscus maniculatus in Algonquin Park, Canada. J Parasitology, v.46, p.373-382, 1960 .
GALVÃO, V.A. Estudos sobre Capiliaria hepatica: uma avaliação do seu papel patogênico para o homem. Mem Inst Oswaldo Cruz, v.76, n.4, p.415-433, 1981.

KOHATSU, H., ZAHA, O., SHIMADA, K., et al. A spaceoccupying lesion in the liver due to Capilaria hepatica infection. Am J Trop Med Hyg, v.52,n.5,p.414-418,1995.

LUTTERMOSER, G.W. An experimental study of Capiliaria hepatica in the rat and the mouse. Am J Hyg, v.27, p.321340,1938

MANDORINO, I., REBOUÇAS, M.M. Hepatic capiliariasis in caititu (Tayassu tajacu). Arq Inst Biol São Paulo, v.58, n.1/2, p.61-62, 1991 .

SANTOS, M.N., BARROS, C.S.L. Capilaria hepatica, parasitismo do cão e gato no Estado do Rio Grande do Sul. Ver Med Vet, São Paulo, v.9, n.2, p. 133-140,1973.

SHORB, D.A. Experimental infestation of white rats with Hepaticola hepatica. J Parasitolgy, v.17, p.151-154,1931.

SILVEIRA, D., NOGUEIRA, R.H.G., SILVA, J.M.L., $\boldsymbol{e} \boldsymbol{t} \boldsymbol{a l}$. Sobre um caso de capilariose hepática em cão. Arq Esc Vet UFMG, Belo Horizonte, v.27, n.2, p.231-234,1975.

SOLOMON, G.B., HANDLEY, C.O. Jr. Capiliaria hepatica (Bancroft, 1893) in Appalachian Mammals. J Parasitology, v.57, n.5, p.1142-1144,1971.

SOULSBY, E.J.L. Helminths, arthropods and protozoa of domesticated animals, $6^{\text {th }}$ ed. London: Baillière Tindall, 1968. The aplasmid nematodes: p.314-325

STOKES, R. Capiliaria hepatica in a dog. Aust Vet J, v.49, n.2 p.109,1973.

TABOADA, J., DIMSKI, D.S. Hepatic encephalopathy: clinicai signs, pathogenesis, and treatment. Vet Clin North Amer: Small Anim Pratice, v.25, n.2, p.337-355,1995.

VIANNA, Y.L. Sobre um caso de capilariose hepática em canino do Rio de Janeiro. Veterinária, v.7, n.2, p.8-20,1954.

WRIGHT, W.H. Hepaticola $\boldsymbol{s}$ p in the liver of dogs. J Parasitol, v.17,p.54-55,1930.

Ciência Rural, v. 30, n. 4, 2000. 